\title{
Unknown Mechanism of Plants Response to Drought: Low Soil Moisture and Osmotic Stresses Induce Severe Decrease in CEC and Increase in Acidity of Barley Roots
}

\author{
Malgorzata Lukowska ${ }^{1}$ \& Grzegorz Jozefaciuk ${ }^{1}$ \\ ${ }^{1}$ Institute of Agrophysics of Polish Academy of Sciences, Lublin, Poland \\ Correspondence: Grzegorz Jozefaciuk, Institute of Agrophysics of Polish Academy of Sciences, Lublin, Poland. \\ Tel: 48-81-744-450-61.E-mail: jozefaci@ipan.lublin.pl
}

Received: July 30, 2013 Accepted: August 20, 2013 Online Published: September 15, 2013

doi:10.5539/jas.v5n10p204 URL: http://dx.doi.org/10.5539/jas.v5n10p204

\begin{abstract}
Huge amount of papers describe plant response to drought, however information on reaction of plant roots cation exchange capacity and surface acidity on draught conditions is up to date lacking. These parameters are important for amount and ratio of cations uptake by plants. Since other stresses induce changes in roots cation exchange capacity and acidity, we hypothesized that draught does this also. In this paper we checked this hypothesis. Surface charge properties of roots of drought tolerant and draught resistant barley varieties taken from pot (soil draught at water potential $\mathrm{pF}=3.5$ ) and hydroponic (osmotic stress induced by mannitol) experiments were determined using back-titration method. The stresses were applied at tillering stage. Soil draught and osmotic stress caused even fivefold decrease in cation exchange capacity and up to tenfold increase in acidity of roots of all barley varieties. Surface charge properties of nonstressed roots did not differentiate draught resistant and draught tolerant varieties, however the intensity of roots reaction on the stresses seemed to be higher for draught sensitive plants. This newly presented mechanism of plant reaction on drought indicates that nutrients uptake by plants can be severely limited and relative uptake of polyvalent cations (aluminum or heavy metals) may increase causing additional toxicity. This may serve as additional explanation of plant growth and yield limitation in dry environments.
\end{abstract}

Keywords: CEC, low moisture stress, osmotic stress, barley, roots, surface acidity, surface charge

\section{Introduction}

Drought is a worldwide problem that threatens food supplies depending on its intensity and duration (Kaya et al., 2006; Wu \& Chen 2013), therefore the response of plants to drought stress has been extensively investigated (Turner et al., 2001; Blum, 2005; De Micco \& Aronne, 2012). At a whole plant level the effects of drought is usually perceived as a decrease in growth, photosynthetic carbon assimilation and water status. The plants evolve a great number of well recognized adaptive mechanisms that allow the biochemical systems to cope with increased water deficit wherein the flexibility of cell metabolism and its fast acclimation to changes in environmental conditions is a first essential step in stress avoidance (Zlatev \& Lidon, 2012). Roots are the first organs of plant which meet the stress and their biomass, length, density and depth are the main drought traits contributing to final yield under drought environments (Subbarao et al., 1995). A most common observation at drought stress is the increase in root/shoot dry matter weight ratio that results from the relatively greater decrease in shoot growth than in root growth. For many plant species the root continues to grow at water potentials that cause complete inhibition of shoot growth. In dry soils roots distribution is deeper and very fine root branches are developed. Root mortality and the normal rate of root turnover may be quite appreciable, as well (Blum, 1996).

From early beginning of plant chemistry cation exchange capacity (CEC) of plant roots is considered to play very important role in cation interchange between plants and soil colloids (Hoagland \& Broyer, 1936; Jenny \& Overstreet, 1939; Fried \& Broeshart, 1967; Marschner, 2012). Cation exchange properties of plant roots result from the presence of a negative charge on root components, developed from dissociation of acidic functional groups of different acidic strengths occurring on various molecules from which the root tissue is composed. The root surface charge changes during various stressing conditions that is most probably associated with changes in roots tissue structure and chemical composition. This was proved for aluminum and heavy metals toxicity by 
Jozefaciuk and Szatanik-Kloc (2004) or Szatanik-Kloc (2010). Therefore we hypothesized that low moisture stress can also affect roots surface charge properties, that to our knowledge still remains to be elucidated.

To do this we applied low moisture stress to barley grown in soil and check changes in roots charge properties. Since extraction of intact roots from soils is extremely difficult and may produce artifacts, to verify findings, results from hydroponic experiment with mannitol-induced osmotic stress were used.

\section{Experiments}

\subsection{Experiment 1: Soil Water Deficit Stress}

\subsubsection{Materials and Methods}

Two barley varieties: Sebastian (Polish) supposed to have small tolerance to water deficit, and Maresi (German) of supposed high tolerance were studied. Personal information on plants tolerance was gained from prof. Maria Surma, Institute of Plant Genetics PAS, Poznan, Poland and from prof. Alicja Pecio, Institute of Soil Science and Plant Cultivation SRI (IUNG), Pulawy, Poland. The seeds of both varieties disinfected by soaking in $1 \%$ sodium hypochloride were placed in six pots containing light loamy soil of water potential adjusted to $\mathrm{pF}=2.2$ that provided optimal soil moisture conditions. Plants growth (10 plants per pot) was continued in $16 \mathrm{~h} \mathrm{light} / 8 \mathrm{~h}$ dark regime providing by yellow sodium light at a temperature of $20 \pm 5^{\circ} \mathrm{C}$ and at $50 \%$ humidity. Everyday the pots were watered to $\mathrm{pF}=2.2$ level. After reaching the tillering stage, in three pots of each variety the watering was stopped until the $\mathrm{pF}=3.5$ (difficultly available water) was reached and such moisture conditions were kept for additional 11 days for the stressed plants. Next the roots of the stressed and the control plants were harvested with special care undertaken to remove the roots from the soil as gently as possible. All harvested roots were rinsed 3 times in 0.01 $\mathrm{mol} \mathrm{dm} \mathrm{m}^{-3} \mathrm{HCl}$ to remove exchangeable basic cations, 5 times in distilled water, air dried and cut onto around $5 \mathrm{~mm}$ length pieces. Roots coming from each pot were collected separately.

The roots were then titrated using Titrino autotitration unit provided by Mettler Toledo equipped with Orion Research combined electrode. The flux of nitrogen through titrated medium was applied to preserve entrance of carbon dioxide from the laboratory air. Back titration procedure (Duquette \& Hendershot, 1993; Nederlof, 1993) with a few modifications was used according to the following description. A suspension containing $0.100 \mathrm{~g}$ of dry roots in $1 \mathrm{~mol} \mathrm{dm}^{-3} \mathrm{NaCl}$ solution was stored overnight and adjusted to $\mathrm{pH} \approx 2.95$ by additions of small increments of $1 \mathrm{~mol} \mathrm{dm}^{-3} \mathrm{HCl}$. After c.a. 5 min equilibration the suspension was titrated using $0.100 \mathrm{~mol} \mathrm{dm}-3 \mathrm{NaOH}$ in $1 \mathrm{~mol}$ $\mathrm{dm}^{-3} \mathrm{NaCl}$ solution with a rate of $30 \mu \mathrm{l} / \mathrm{min}$. The amount of the titer consumed between $\mathrm{pH} 3$ to 10 was recorded with a step of $0.2 \mathrm{pH}$ unit. The titration curve of the suspension medium $\left(1 \mathrm{~mol} \mathrm{dm}^{-3} \mathrm{NaCl}\right.$ solution) was determined in similar way. For roots taken from each of 3 pots, the measurements were performed in three replicates with the deviation not exceeding $6 \%$, however the differences between pots were up to $19 \%$. This is important to note that in the above procedure the equilibrium conditions are not reached, so the titration curves can be used rather for comparative purposes.

The base consumed during the titration by the root suspension, $N_{\text {susp }}$, is consumed by acids located on root surface, $N_{s}$, and by acids present in the solution, $N_{\text {sol }}$ :

$$
N_{\text {susp }}=N_{s}+N_{\text {sol }}
$$

The $N_{s}$ hydroxyls are used for neutralization of acidic groups, $\mathrm{AH}$, bound to the root tissue surface, $\mathrm{S},(\mathrm{SAH})$ in the reaction:

$$
\mathrm{SAH}+\mathrm{OH}^{-}=\mathrm{SA}^{-}+\mathrm{H}_{2} \mathrm{O} .
$$

This reaction increases the $\mathrm{pH}$ and produces negatively charged surface anion, $\mathrm{SA}^{-}$, thus increasing variable surface charge, $Q_{V}$, of the roots.

Under some assumptions (Nederlof, 1993; Jozefaciuk \& Szatanik-Kloc, 2004), the $N_{s}$ versus $\mathrm{pH}$ dependence may be treated as the $Q_{V}$ versus (apparent) surface dissociation constant, $\mathrm{p} K_{\text {app }}$, dependence:

$$
N_{S}(p H)=Q_{v}(p H)=Q_{v}\left(p K_{\text {app }}\right), \quad p K_{\text {app }}=p H
$$

Assuming that at the beginning of the titration the $Q_{V}$ equals zero, the maximal value of $N_{s}$ measured within the whole experimental window, $N_{\max }$, is equivalent to total surface charge of the roots, $Q v_{\text {tot }}$. Following the most common method for root CEC estimation based on titration of acid washed roots to $\mathrm{pH}=7$ (Williams \& Coleman, 1950; Heintze, 1964), the $N_{s}$ at $\mathrm{pH}=7$ was considered as the roots CEC. 
From the function described by Eq 2 the (normalized) distribution function of apparent surface dissociation constants, $f\left(\mathrm{p} K_{a p p}\right)$, was estimated by finding the first derivative of $Q_{V}\left(\mathrm{p} K_{a p p}\right)$ on $\mathrm{p} K_{a p p}$ divided by $N_{\max }$ :

$$
f\left(p K_{a p p}\right)=1 / N_{\max } d Q_{v}\left(p K_{a p p}\right) / d p K_{a p p}
$$

The average value of $\mathrm{p} K_{a p p}, \mathrm{p} K_{a p p, a v}$, was calculated as:

$$
p K_{a p p, a v}=\int p K_{a p p} f\left(p K_{a p p}\right)
$$

which is a direct measure of the average energy of the proton binding by the studied surface.

\subsubsection{Results}

$Q_{V}$ vs. $\mathrm{pH}$ dependencies for the studied roots are presented in Figure 1. The average curves are presented for each treatment and variety.

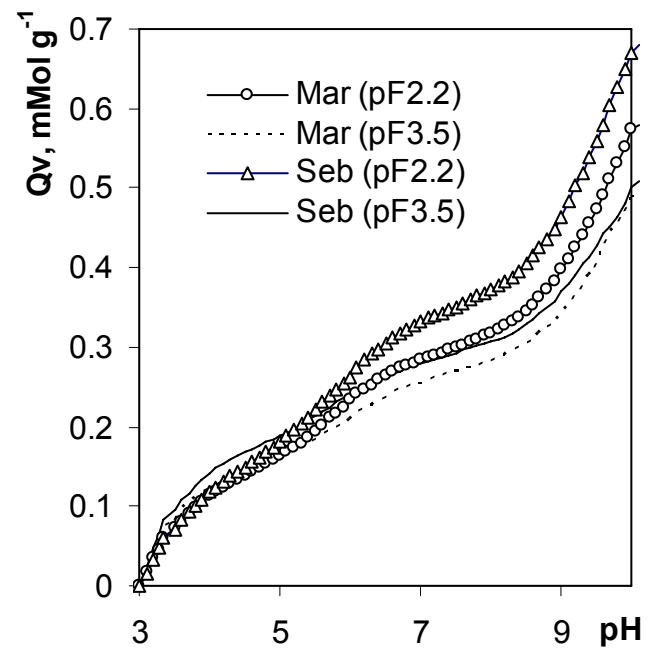

Figure 1. Dependence of variable surface charge of roots of barley var. Sebastian (Seb) and Maresi (Mar) on pH in relation to soil moisture. The number in parentheses denotes the soil $\mathrm{pF}$ value

The roots taken from low moisture soil have markedly lower variable charge than these taken from optimum moisture soil. In low $\mathrm{pH}$ range higher amount of charge is developed on the stressed roots and in high $\mathrm{pH}$ range the variable charge seems to increase slower than this of the control roots. This indicates that the relative amounts of surface acidic groups also change under the stress that is seen from distribution functions of $\mathrm{p} K_{\text {app }}$ of the roots presented in Figure 2.

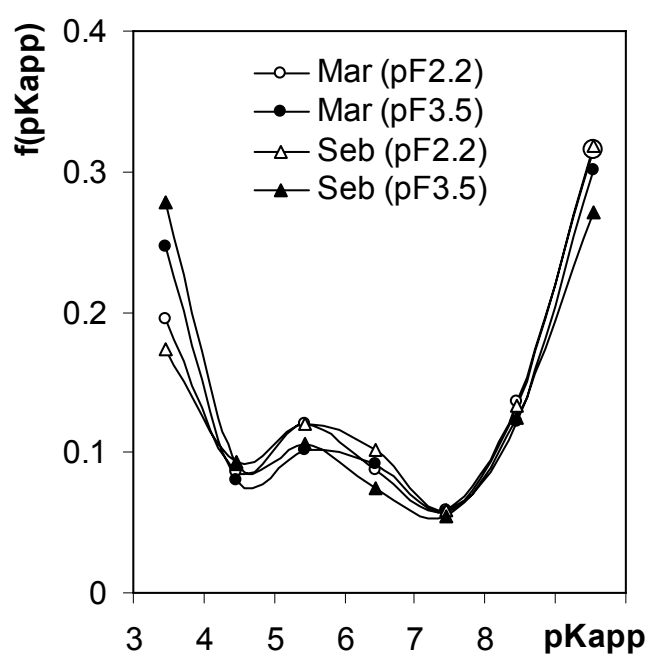

Figure 2. Distribution function of apparent surface dissociation constants of roots of barley var. Sebastian and Maresi in relation to soil moisture. Abbreviations as in Figure 1 
For all roots these functions are $\mathrm{W}$-shaped with maximum amounts of charge-generating groups having the lowest and the highest $\mathrm{p} K_{a p p}$ values and smaller, well developed peak at intermediate $\mathrm{p} K_{a p p}$ values is present. Soil drought induces an increase in the amount of strongly acidic groups and a decrease in the amount of very weak acidic groups.

We feared that the observed differences in surface charge properties of the control and the stressed roots may be not necessarily due to different growing conditions but could be also affected by root harvesting procedures. The finest roots may be adhered to soil grains and removed with the soil at harvesting and some finest soil components adhered to the roots may be taken together with the experimental material. Therefore we performed the next experiment that allowed for observations of roots charge in more clear environments with limited water supply.

\subsection{Experiment 2: Osmotic Stress in Hydroponic Culture}

\subsubsection{Materials and Methods}

The disinfected seeds of two previously used barley varieties Sebastian and Maresi, and two others: Stratus (Polish, drought sensitive) and CamB1 (Syrian, drought tolerant) were germinated on moist tissue paper in dark room at $20^{\circ} \mathrm{C}$. Three day old seedlings of each variety were transformed to 12 vessels with Hoagland nutrient solution and the further plants growth (10 plants per vessel), was continued at the same external conditions as in pot experiment. After reaching tillering stage (14 days) different concentrations of mannitol: 0 (control), 0,025; 0,05 and 0,075 mol $\mathrm{dm}^{-3}$ were added to every 3 vessels and all plants were grown for next 7 days. The harvested roots were subjected to the same procedures as the soil-grown roots. For roots taken from each of 3 vessels, the titration curves measurements were performed in three replicates with the deviation not exceeding $4 \%$. The differences between roots coming from vessels of that same stress did not exceed $10 \%$ and they were significantly smaller than those for soil-grown roots.

\subsubsection{Results}

Exemplary variable charge vs. pH dependencies for Maresi and Sebastian roots are presented in Figures 3 and 4, respectively.

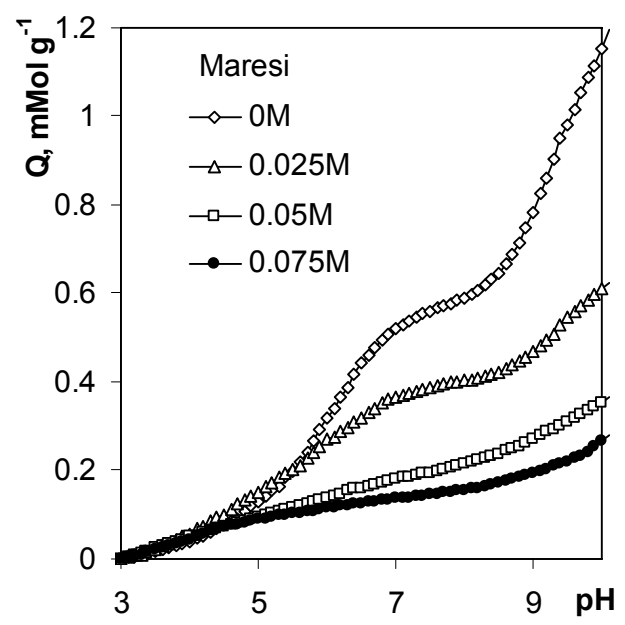

Figure 3. Dependence of variable surface charge of roots of barley var. Maresi on $\mathrm{pH}$ in relation to osmotic stress intensity in hydroponic culture. Abbreviations: $\mathrm{M}$ addition of mannitol. The number before $\mathrm{M}$ denotes mannitol molar concentration 


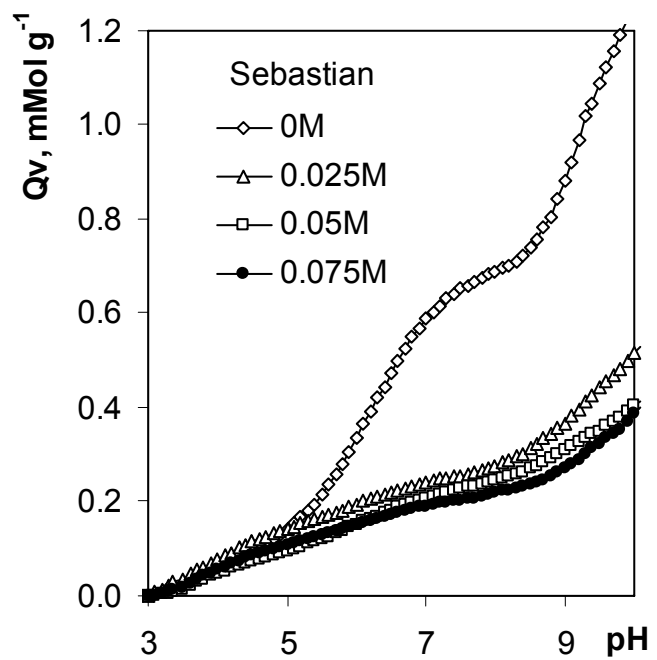

Figure 4. Dependence of variable surface charge of roots of barley var. Sebastian on $\mathrm{pH}$ in relation to osmotic stress intensity. Abbreviations as in Figure 3

Similarly to soil drought osmotic stress induces consecutive decrease of the $Q v$ of all roots. From distribution functions of $\mathrm{p} K_{a p p}$ of the same roots presented in Figures 5 and 6 one can see that the control roots exhibit well developed peak of surface groups of weak acidity $\left(\mathrm{p} K_{a p p}\right.$ around 6.0) and next peak at high $\mathrm{p} K_{\text {app }}$ values reflecting high amount of very weak acidic groups. Osmotic stress induces an increase in the amount of strongly acidic groups.

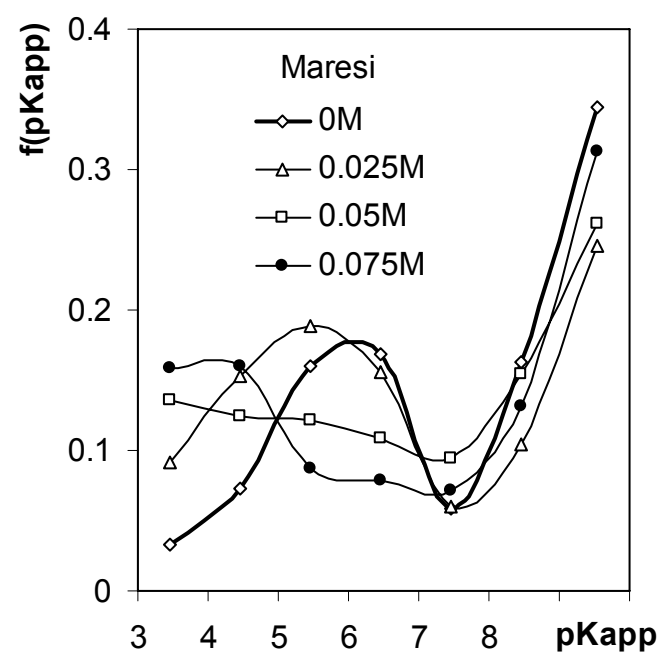

Figure 5. Distribution function of apparent surface dissociation constants of roots of barley var. Maresi in relation to osmotic stress intensity. Abbreviations as in Figure 3 


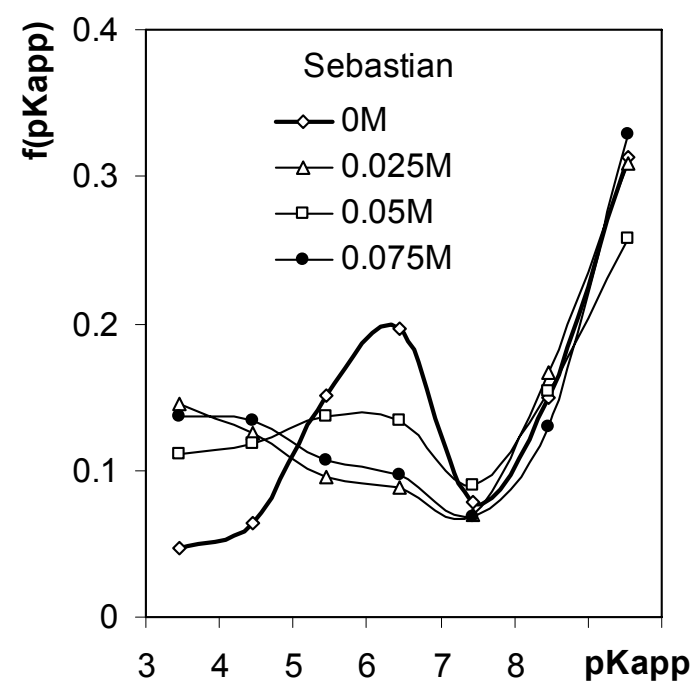

Figure 6. Distribution function of apparent surface dissociation constants of roots of barley var. Sebastian in relation to osmotic stress intensity. Abbreviations as in Figure 3

\section{Discussion}

The $\mathrm{p} K_{\text {app }}$ distribution functions are different for roots taken from soil and from hydroponic cultures. Roots coming from soil have markedly higher amount of strongly acidic functional groups. These groups may occur on root hairs, as far as roots grown in soil produce more hairs than these grown in water, however these may also come from soil material adhered to the roots.

Values of $Q v_{t o t}, \mathrm{CEC}$ and $\mathrm{p} K_{a p p, a v}$ of the control roots taken from hydroponic cultures and from soil are presented in Table 1. No marked differences in the estimated charge parameters are observed for all varieties grown in hydroponic cultures thus drought tolerant and drought sensitive plants are not distinguishable. Despite surface charge parameters estimated for soil-grown roots are smaller, no marked differences between drought tolerant Maresi and drought sensitive Sebastian are seen, as well.

Table 1. Values of total variable charge, cation exchange capacity and negative logarithms of apparent surface dissociation constants of the studied control roots

\begin{tabular}{lllllll}
\hline & Seb & Strat & Cam & Mar & Seb(S) & Mar(S) \\
\hline Qtot, mMole g-1 & $1.28 \pm 0.052$ & $1.20 \pm 0.049$ & $1.33 \pm 0.054$ & $1.19 \pm 0.046$ & $0.68 \pm 0.056$ & $0.58 \pm 0.047$ \\
CEC, mMole g-1 & $0.59 \pm 0.021$ & $0.58 \pm 0.024$ & $0.60 \pm 0.024$ & $0.52 \pm 0.019$ & $0.33 \pm 0.027$ & $0.28 \pm 0.023$ \\
pKapp & $7.23 \pm 0.47$ & $7.12 \pm 0.44$ & $7.27 \pm 0.38$ & $7.20 \pm 0.51$ & $6.84 \pm 0.84$ & $6.82 \pm 0.91$
\end{tabular}

Description: First four columns show data for plant varieties grown in hydroponic cultures with no mannitol addition whereas varieties abbreviated with ( $\mathrm{S}$ ) were grown in soil at $\mathrm{pF}=2.2$. After \pm sign shown is the standard deviation of 3 average values, each estimated from individual experimental replicate of control plants (3 hydroponic vessels or 3 soil pots).

Effect of both stresses on root surface charge parameters is illustrated in Figures 7-9. In these figures, the ratio of a value of a given charge parameter of the stressed roots to its value for the control root is presented on y-axis. 


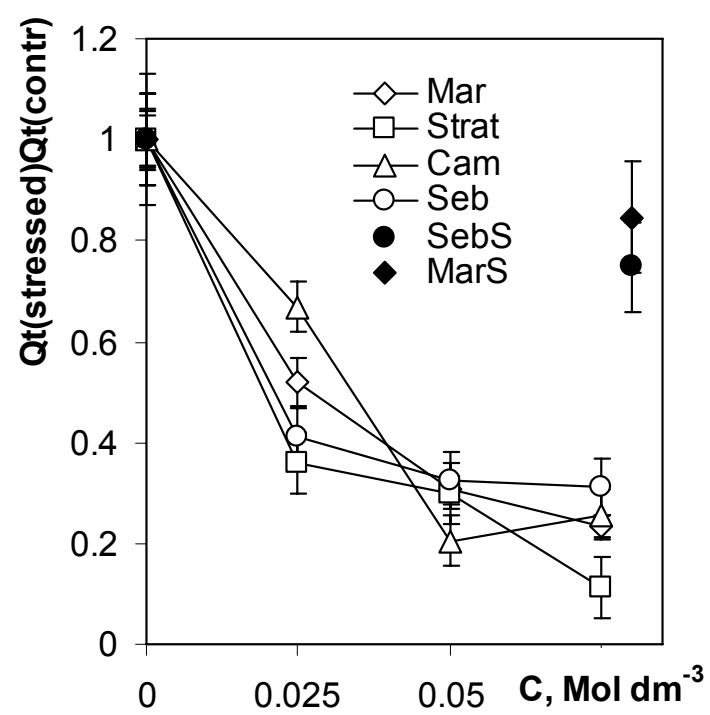

Figure 7. Relative changes in total surface charge of the studied roots at various growing conditions. On y-axis the ratio of the charge of the roots grown at osmotic or low soil moisture stress to the charge of the control roots is given. Abbreviations: empty points denote roots grown in hydroponic culture with addition of mannitol of concentration shown on $\mathrm{x}$-axis. Here the control roots (points at $\mathrm{C}=0$ ) were grown without mannitol addition. Black points denote plants grown in soil $(\mathrm{S})$. Here the control roots were grown at $\mathrm{pF} 2.2$ and points located at $\mathrm{C}=0.8$ denote soil drought conditions $(\mathrm{pF}=3.5)$. Varieties of barley $\mathrm{Seb}-$ Sebastian, Strat - Stratus, $\mathrm{Cam}-\mathrm{CamB} 1$ an Mar - Maresi. Error bars show standard deviations between 3 average values, each estimated from individual experimental replicate of plants growth (3 hydroponic vessels or 3 soil pots)

The marked decrease in $Q v_{t o t}$ and CEC due to the stresses is observed (Figures 7 and 8, respectively), particularly at osmotic stress of highest mannitol concentrations. Decrease in CEC may drastically limit cationic nutrients uptake under drought conditions. The decrease in negative logarithms of average surface dissociation constants $\left(\mathrm{p} K_{a p p}\right)$ presented in Figure 9 indicates increase in root surface acidity under both stresses. The resulting higher protons dissociation produces more charged groups on the root surface at a given external soil conditions that may induce higher surface electric potential. At higher potentials polyvalent cations are attracted better than monovalent ones thus a danger of aluminum and heavy metals toxicity may increase simultaneously. From the second side, higher surface potential can induce higher water (strong dipole) binding forces what can decelerate water lost and help plant to compete with soil for water. This is worth mentioning that aluminum and heavy metals toxicity also induce decrease in roots CEC, however the surface acidity decreases (Jozefaciuk \& Szatanik-Kloc, 2004; Szatanik-Kloc, 2010). This seems logic because lower surface acidity decreases toxic cations uptake. Usually root surface charge is positively correlated with its surface area (Szatanik-Kloc, 2010), so a decrease in surface area at drought is likely to occur. This can further diminish the intensity of water lost by roots, however simultaneously the further water uptake can be reduced also. Finding how root surface area and energy of water binding change under drought seems interesting and we plan to perform such studies. 


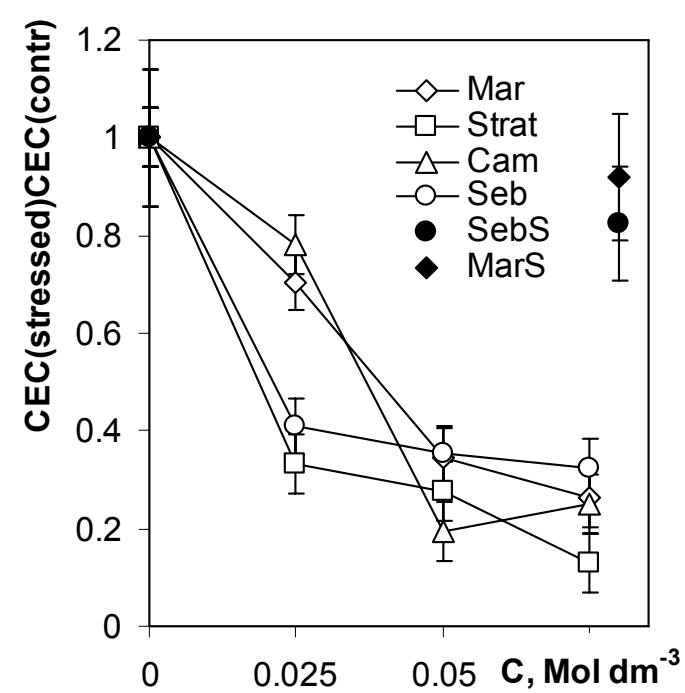

Figure 8. Relative changes in cation exchange capacity of the studied roots at various growing conditions. Abbreviations and comments as in Figure 3

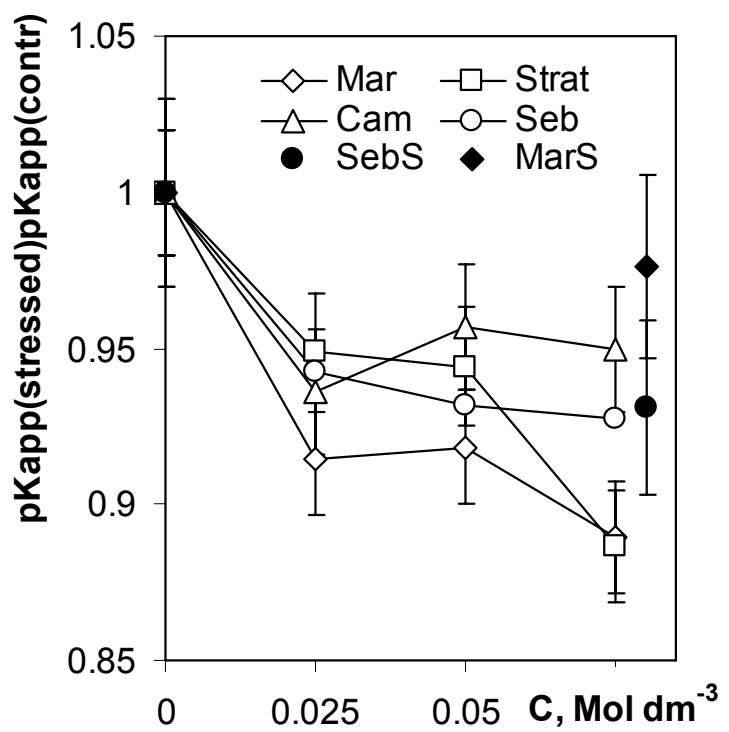

Figure 9. Relative changes in negative logarithms of apparent surface dissociation constants of the studied roots at various growing conditions. Abbreviations and comments as in Figure 3

The intensity of changes in variable charge parameters of the roots under low soil moisture and osmotic stresses described above may reflect the drought-tolerance of the studied barley varieties, as far as the most intensive changes are observed for drought sensitive Sebastian and Stratus and the least intensive for drought tolerant Maresi and CamB1. Differences in roots CEC under osmotic stress at the lowest mannitol concentration best distinguish both plants groups. To validate this observation studies of more barley varieties and other plants are necessary.

At present we did not attempt to look for the eventual connections of changes in variable charge properties and root tissue composition under the stresses. These may be due to a damage of the cell walls and the outflow of the charged components from the cell interior, decrease in the amount of (charged) root hairs and/or any other changes in cell walls chemistry caused by partial root necrosis or growing of new roots of other composition.

Interesting is also how long changes in roots surface charge persist after the end of a drought i.e. how long plants suffer drought induced nutrition hunger. This will be subject of our future studies. 


\section{Conclusions}

Low soil moisture and osmotic stresses decreased variable charge of the studied barley roots, even down to around $20 \%$ of the values found for nonstressed plants. Parallel increase in average values of surface dissociation constants of roots surface acidic groups from around $10^{-7}$ to around $10^{-6}$ was noted, indicating around tenfold rise in roots surface acidity. The intensities of the above changes and not the surface charge characteristics of nonstressed roots apparently reflect the tolerance of barley varieties against drought.

\section{Acknowledgements}

This work was supported by the European Regional Development Fund through the Innovative Economy Program for Poland 2007-2013, project WND-POIG.01.03.01-00-101/08 POLAPGEN-BD. Biotechnological tools for breeding cereals with increased resistance to drought". The project is realized by POLAPGEN Consortium coordinated by Institute of Plant Genetics, Polish Academy of Sciences in Poznan. Further information about the project can be found at www.polapgen.pl.

\section{References}

Blum, A. (1996). Crop responses to drought and the interpretation of adaptation. Plant Growth Regul. 20, 135-148. http://dx.doi.org/10.1007/BF00024010

Blum, A. (2005). Drought resistance, water-use efficiency, and yield potential-are they compatible, dissonant, or mutually exclusive? Australian J. Agric. Res., 56, 1159-1168. http://dx.doi.org/10.1071/AR05069

De Micco, V., \& Aronne, G. (2012) Morpho-Anatomical Traits for Plant Adaptation to Drought. In R. Aroca (Ed.), Plant Responses to Drought Stress (pp. 37-61). Verlag Berlin Heidelberg: Springer. Retrieved from http://dx.doi.org/10.1007/978-3-642-32653-0_2

Duquette, M., \& Hendershot, W. (1993) Soil surface charge evaluation by back-titration, I. Theory and method $\begin{array}{lllllll}\text { development. Soil } & \text { Sci. } & \text { Soc. } & \text { Am. } & \text { J., } & 57, & 1222-1228 \text {. }\end{array}$ http://dx.doi.org/10.2136/sssaj1993.03615995005700050011x

Fried, M., \& Broeshart, H. (1967). The Soil - Plant System in relation to inorganic nutrition. New York, San Francisco: Academic Press.

Heintze, S. G. (1964). Studies on cation exchange capacities of roots. Plant and Soil, 4, 365-383.

Hoagland, D. R., \& Broyer, T. C. (1936) General nature of the process of salt accumulation by roots with description of experimental methods. Plant Physiol., 11, 471-507. http://dx.doi.org/10.1104/pp.11.3.471

Jenny, H., \& Overstreet, R., (1939) Cation interchange between plant roots and soil colloids. Soil Sci., 54, 177-189.

Jozefaciuk, G., \& Szatanik-Kloc, A. (2004) Decrease in variable charge and acidity of root surface under Al treatment are correlated with Al tolerance of cereal plants. Plant Soil, 260, 137-145. http://dx.doi.org/10.1023/B, PLSO.0000030175.23904.5a

Kaya, M. D., Okcub, G., Ataka, M., Cikılic, Y., \& Kolsaricia, O. (2006) Seed treatments to overcome salt and drought stress during germination in sunflower (Helianthus annuus L.), Eur. J. Agron., 24, 291-295. http://dx.doi.org/10.1016/j.eja.2005.08.001

Marschner, P. (Ed.). (2012). Marschner's Mineral Nutrition of Higher Plants. Elsevier, London. UK.

Nederlof, M. M., De Wit, J. C., Riemsdijk, W. H., \& Koopal, L. K. (1993) Determination of proton affinity distributions for humic substances. Environmental Sci. Technol., 27, 846-856. http://dx.doi.org/10.1021/es00042a006

Subbarao, G. V., Johansen, C., Slinkard, A. E., Rao R. C. N., Saxena, N. P., \& Chauhan, Y. S. (1995) Strategies and scope for improving drought resistance in grain legumes, Crit. Rev. Plant Sci., 14, 469-523.

Szatanik-Kloc, A. (2010) Changes in surface properties of monocotyledonous and dicotyledonous plants determined by aluminum and copper toxicity. Acta Agrophysica, 176, 1-183 (in Polish).

Turner, N. C., Wright, G. C., \& Siddique, K. H. M. (2001) Adaptation of grain legumes (pulses) to water-limited environments. Adv. Agron., 71, 123-231. http://dx.doi.org/10.1016/S0065-2113(01)71015-2

Williams, D. E., \& Coleman, N. T. (1950) Cation exchange properties of plant root surfaces. Plant and Soil, 2, 243-256. http://dx.doi.org/10.1007/BF01852352 
Wu, C., \& Chen, J. M. (2013) Diverse responses of vegetation production to interannual summer drought in North America. International Journal of Applied Earth Observation and Geoinformation, 21, 1-6. http://dx.doi.org/10.1016/j.jag.2012.08.001

Zlatev, Z., \& Lidon, F. J. C. (2012) An overview on drought induced changes in plant growth, water relations and photosynthesis. Emir. J. Food Agric., 24(1), 57-72.

\section{Copyrights}

Copyright for this article is retained by the author(s), with first publication rights granted to the journal.

This is an open-access article distributed under the terms and conditions of the Creative Commons Attribution license (http://creativecommons.org/licenses/by/3.0/). 